\title{
A perfectly current matched charge pump with wide dynamic range for ultra low voltage applications
}

\author{
Dongdong Zhong ${ }^{1}$, Yan Han ${ }^{1 a)}$, Jun Sun ${ }^{1}$, Qian Zhou ${ }^{1}$, \\ Ray C. C. Cheung ${ }^{2}$, and Wenquan Sui $^{3}$ \\ ${ }^{1}$ Institute of Microelectronics and Optoelectronics, Zhejiang University, \\ Zheda Road 38, Hangzhou 310027, China \\ ${ }^{2}$ Department of EE, City University of Hong Kong \\ ${ }^{3}$ Institute of Nano Research, Zhejiang University \\ a)hany@zju.edu.cn
}

\begin{abstract}
A simple and perfectly current matched charge pump for ultralow voltage applications in phase-locked loops is presented. The charge pump is based on a gate switching architecture to ensure the output dynamic range. The specially designed dual compensation circuits using high threshold transistors together with regular threshold transistors ensure the current mismatch below $1.5 \%$ while keeping the output voltage ranging from 0.02 to $0.76 \mathrm{~V}$ in $40 \mathrm{~nm}$ CMOS Mixed-signal technology at a $0.8 \mathrm{~V}$ supply. And the additional body bias circuit reduces the negative impact from the process variation. The proposed circuit helps PLLs reduce the phase offset and spurs even at ultra low supply voltage.
\end{abstract}

Keywords: charge pump, current match, dynamic range, low voltage, PLL Classification: Electron devices, circuits, and systems

\section{References}

[1] M. Jalalifar and G.-S. Byun: Electron. Lett. 49 (2013) 1436. DOI:10.1049/el.2013. 1304

[2] N. T. Hieu, T.-W. Lee and H.-H. Park: Lasers and Electro-Optics - Pacific Rim (2007) 1. DOI:10.1109/CLEOPR.2007.4391088

[3] H. R. E. Jazi and N. Ghaderi: IEICE Electron. Express 11 (2014) 20130934. DOI:10.1587/elex.10.20130934

[4] M.-S. Hwang, J. Kim and D.-K. Jeong: Electron. Lett. 45 (2009) 135. DOI:10.1049/ el:20092727

[5] N. Joram, R. Wolf and F. Ellinger: Electron. Lett. 50 (2014) 661. DOI:10.1049/el. 2014.0804

[6] C. Yu, M. Kim, H. Kim and Y. Yang: Adv. Mater. Res. 457 (2012) 1178. DOI:10. 4028/www.scientific.net/AMR.457-458.1178

[7] P. Liu, P. Sun, J. Jung and D. Heo: Electron. Lett. 48 (2012) 16. DOI:10.1049/el. 2011.2835

[8] C.-L. Ti, Y.-H. Liu and T.-H. Lin: IEEE International Symposium on Circuits and Systems (2008) 1728. DOI:10.1109/ISCAS.2008.4541771 


\section{Introduction}

Charge pump phase-locked loops (CP-PLLs) are widely used in modern wireless communication systems because of their high speed, low jitter and wide locking range. The CP circuit plays a very important role in a CP-PLL, and its main task is to convert the pulsed output signal of the phase frequency detector (PFD) into an analog voltage signal loaded on the loop filter (LF). However, there are some nonideal factors of $\mathrm{CP}$, bringing about negative effects in the entire loop. Charge/ discharge current mismatch in CP causes output voltage fluctuation, which generates phase offset and reference spurs in the PLL output spectrum [1].

For ultra-low voltage applications, the charge pumps become more difficult to get desired performance, especially the charge/discharge current mismatch and the output voltage headroom. In order to ensure the wide operating range of PLL, a wide dynamic range of the $\mathrm{CP}$ is necessary. In $[2,3]$ specially designed cascade structures are introduced to reduce current mismatch, but the output dynamic range is occupied seriously while at low supply voltage. The active feedback circuits based on operational amplifiers are proposed in $[4,5,6]$ to compensate the current mismatch. However the circuits are so complicated and still sacrifice the output dynamic range. Besides, the operational amplifier itself may not work under ultra low voltage. In [7] an adaptive body bias charge pump circuit is introduced, which uses a number of resistances to compensate current variation. However these resistances can be easily affected by multiple factors such as process variation. Authors in [8] propose a charge pump with relatively simple dual compensation circuits for matching enhancement, but the result isn't so satisfactory.

In this letter, the proposed charge pump operating at $0.8 \mathrm{~V}$ improves current matching with special dual compensation circuits while keeping the output dynamic range nearly rail-to-rail. And the additional body bias circuit reduces the negative impact from the process variation.

\section{Circuit design}

\subsection{Conventional charge pump circuit}

Fig. 1 shows the conventional CP based on a gate switching architecture which is usually used in low voltage area to ensure the output voltage range. However, as the output voltage varies, the charge and discharge currents deviate from each other because of the channel length modulation effect.
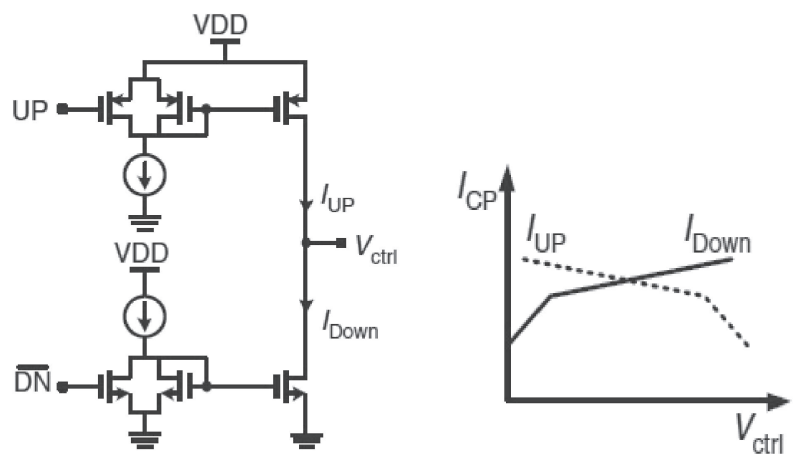


\subsection{Proposed charge pump circuit}

The proposed CP circuit is shown in Fig. 2, which is designed with the purpose to minimize the current mismatch at a low supply voltage while keeping the output dynamic range wide.

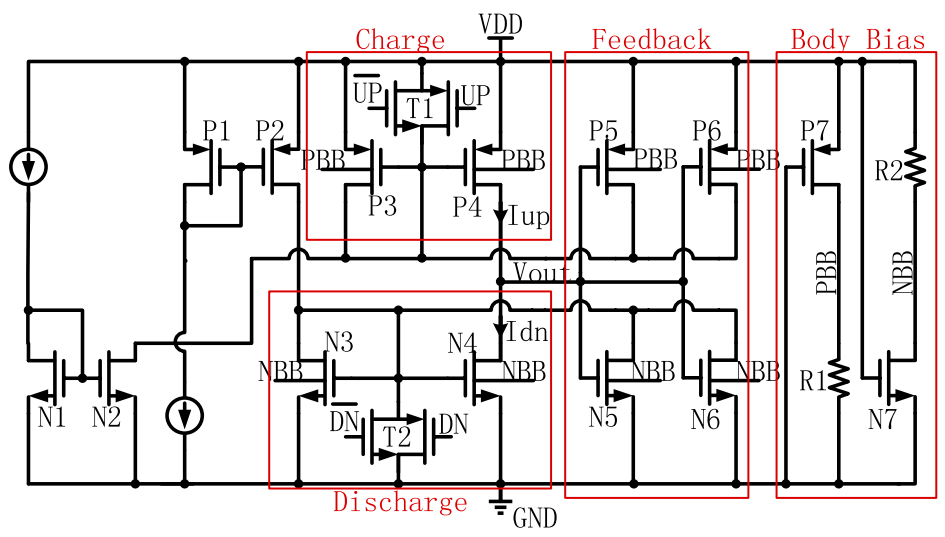

Fig. 2. Proposed charge pump with feedback loop and body bias circuit

This CP utilizes two negative feedback circuits to suppress the current mismatch when the output voltage changes between the supply voltage and the ground potential. In order to adjust the charge and discharge currents more flexibly and accurately, we use a high threshold transistor P6 together with a regular threshold transistor P5 to control the charge current, and a high threshold transistor N6 together with a regular threshold transistor N5 to control the discharge current, while the other transistors are all low threshold transistors for ultra-low voltage application. When the output voltage is close to VDD, N5 and N6 turn on successively, pulling down the gate voltage of N4, thereby reducing the discharge current Idn to match the low charge current Iup. Similarly, when the output voltage is close to GND, P5 and P6 turn on successively, pulling up the gate voltage of P4, thereby reducing the charge current Iup to match the low discharge current Idn. The absolute value of Icp is mainly determined by the current source, while the feedback transistors with two kinds of thresholds are used to produce an accurate matching between charge and discharge currents.

In order to reduce the turn on time of the CP caused by the gate capacitance of the charge/discharge transistors, we use transmission gate instead of single MOS transistor as charge/discharge control switch to increase drive capability, which also helps reduce the charge injection and improve the linearity of the switch. Note that, this $\mathrm{CP}$ is based on a gate switching architecture so that the output dynamic range can be maximized.

However, the output resistance of this structure is relatively small, current match may be degenerated because of the process variation. And in ultra-low voltage applications, it is quite difficult to use cascode structure or feedback opamp to reduce current variation, while keeping enough output dynamic range. This problem also exists in [1]. Therefore, an additional body bias circuit consisting of P7, N7, R1, R2 is employed. The PMOS body bias voltage PBB connects to the bodies of P3, P4, P5, P6, and the NMOS body bias voltage NBB connects to the 
bodies of N3, N4, N5, N6. The forward body bias makes P4, N4 easier to turn on and occupy lower voltage drop while in low supply voltage applications.

To keep balance and get enough adjustment range, we set the initial value at $t \mathrm{t}$ corner of PBB and NBB both at about $400 \mathrm{mV}$ (half the supply voltage) by adjusting the related transistors and resistors. What's more, at ultra-low voltage $(800 \mathrm{mV})$, the $400 \mathrm{mV}$ body bias voltage can make sure that the breakdown of transistors won't happen. And the noise after applying the forward body bias technique will be eliminated by the low pass filter after the CP in PLL. When the process is gradually approaching ss (slow-slow), the body bias voltage PBB drops and NBB rises, which implement forward body bias on the target transistors. Similarly, when the process is gradually approaching ff (fast-fast), the body bias voltage PBB rises and NBB drops, which implement reverse body bias on the target transistors. The same effects occur in fs (fast-slow) and sf (slow-fast). This kind of negative feedback modulation prevents the currents of target transistors from decreasing in slow corner or increasing in fast corner, so that the current match can be conserved. In most references, the process variation is avoided to be discussed for the mismatch can be relatively large when the PMOS charge transistors and NMOS discharge transistors are at different process corners. Here we utilize the body bias circuit to reduce this problem to some extent.

\section{Simulation results}

The proposed CP is implemented in $40 \mathrm{~nm}$ CMOS Mixed-signal technology at a $0.8 \mathrm{~V}$ supply. The simulated charge and discharge currents against the CP output voltage is shown in Fig. 3. The peak current of the $\mathrm{CP}$ is $125 \mathrm{uA}$. The current mismatch is below $1.5 \%$ over the output voltage range from 0.02 to $0.76 \mathrm{~V}$. Fig. 4 shows the output voltage transient simulation result of this $\mathrm{CP}$ with a $50 \%$ duty 20 ns cycle square wave control signal. In Fig. 5, the 1000-case Monte Carlo simulations of maximum current mismatch against process variation are demonstrated. We can see the average current mismatch is reduced from $3.31 \mathrm{uA}$ to $2.46 \mathrm{uA}$, and the standard deviation is reduced from $2.49 \mathrm{uA}$ to $1.87 \mathrm{uA}$ in the 1000 cases. The mismatch cases larger than $5 \mathrm{uA}$ which mainly occur at sf and fs process corners are reduced from $240 / 1000$ to $105 / 1000$, less than $50 \%$ of before. In Fig. 6, the current mismatches at peak current due to voltage/temperature variations are presented. We can see that at the voltage variation of $-10 \% \sim+10 \%$ supply

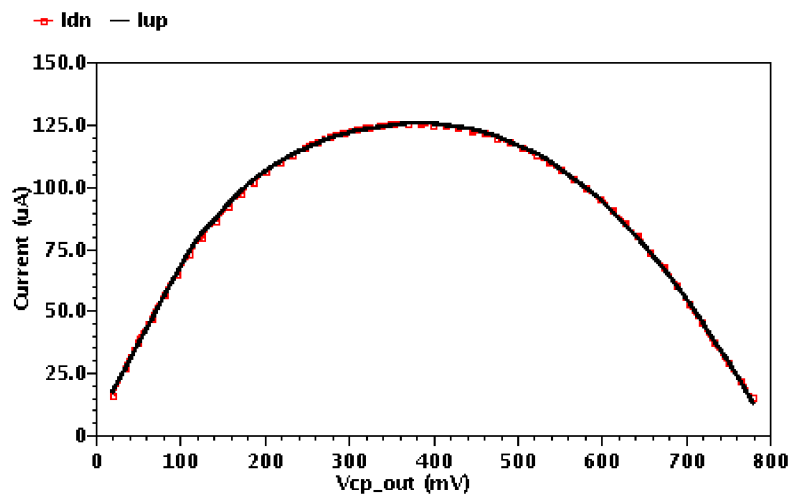

Fig. 3. Simulated charge/discharge currents against CP output voltage 


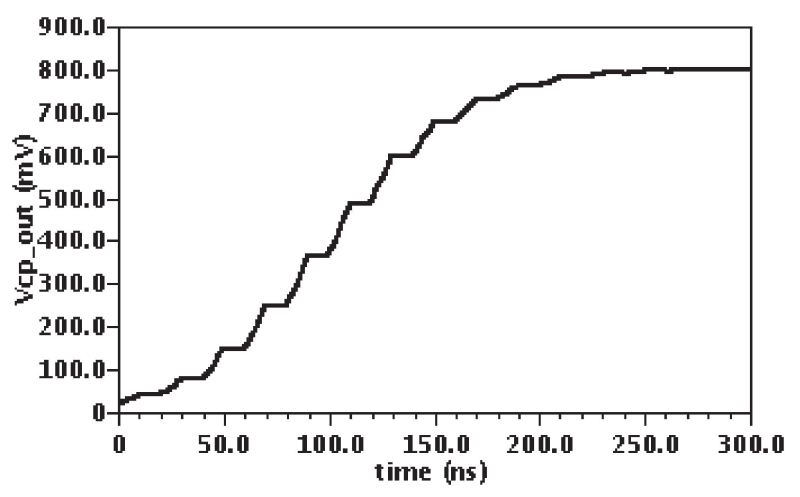

Fig. 4. Simulated output voltage of $\mathrm{CP}$ in charging procedure
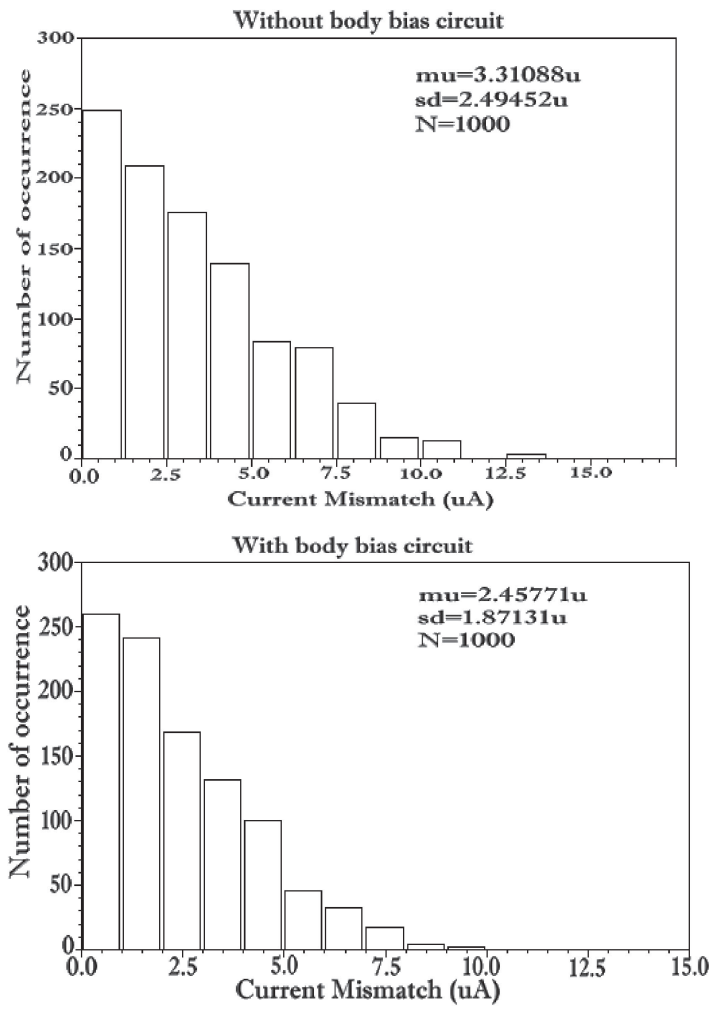

Fig. 5. Monte Carlo simulations of maximum current mismatch
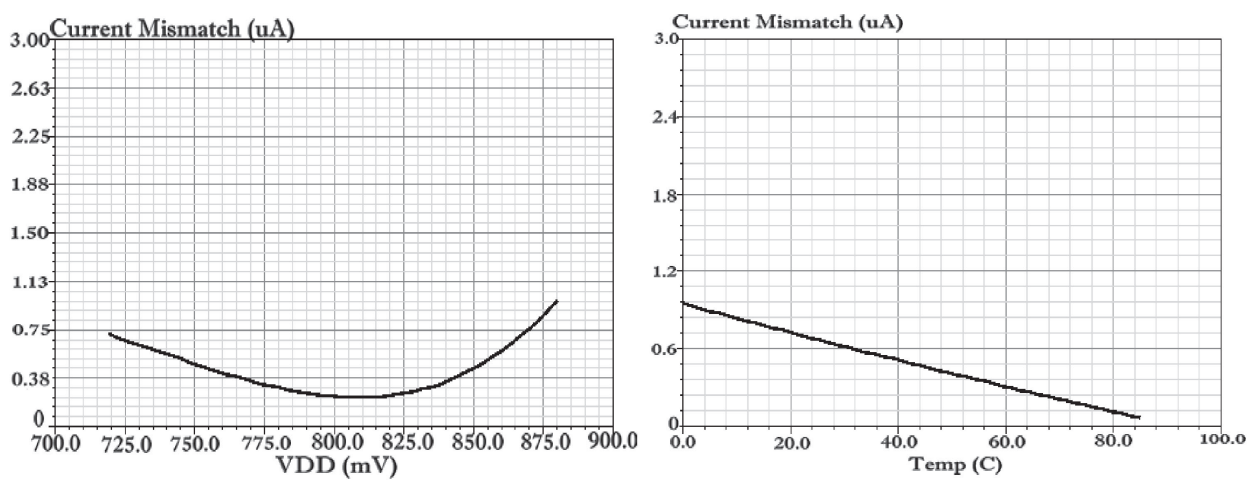

Fig. 6. Current mismatches of voltage/temperature variations 


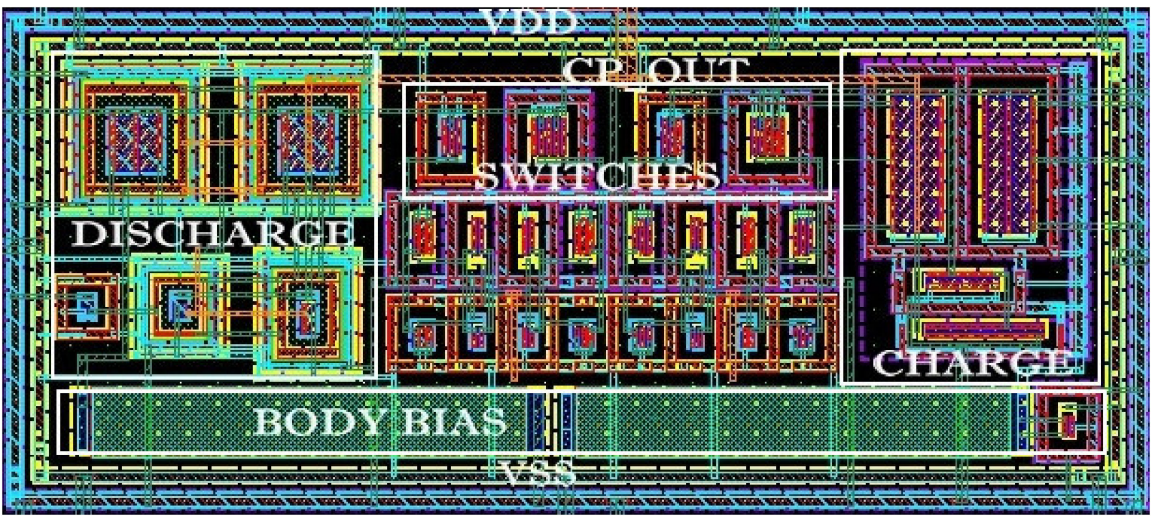

Fig. 7. Layout of the proposed CP

Table I. Performance comparison

\begin{tabular}{|c|c|c|c|c|c|}
\hline & {$[3]$} & {$[4]$} & {$[6]$} & {$[7]$} & This work \\
\hline Technologies (um) (CMOS) & 0.18 & 0.13 & 0.13 & 0.13 & 0.04 \\
\hline Supply voltage (V) & 1.8 & 1.2 & 1.2 & 1.2 & 0.8 \\
\hline CP current (uA) & 50 & 100 & 225 & 140 & 125 \\
\hline Current mismatch (\%) & N/A & $<3.2$ & $<4.9$ & N/A & $<1.5$ \\
\hline $\begin{array}{c}\text { Output voltage range } \\
(\mathrm{V}, \%)\end{array}$ & $0.3-1.58$ & $0.2-1.0$ & $0.2-1.0$ & $0.2-1.0$ & $0.02-0.76$ \\
\cline { 2 - 6 } & 71 & 67 & 67 & 67 & 93 \\
\hline
\end{tabular}

voltage and the temperature variation of $0 \sim 85^{\circ} \mathrm{C}$, the current mismatches are both less than $1 \mathrm{uA}$, for it isn't like the process variation which has sf and fs situation making the charge/discharge transistors change to the opposite directions. And Fig. 7 shows the layout of the proposed CP. Table I compares the post layout simulation results of the designed circuit to recent publications. The performance of the current mismatch and output voltage range are both superior.

\section{Conclusion}

A simple and perfectly current matched charge pump for PLLs is proposed. The specially designed dual compensation circuits using high threshold transistors together with regular threshold transistors ensure the current mismatch below $1.5 \%$ while keeping the output voltage ranging from 0.02 to $0.76 \mathrm{~V}$ in $40 \mathrm{~nm}$ CMOS Mixed-signal technology at a $0.8 \mathrm{~V}$ supply. And the additional body bias circuit reduces the negative impact from the process variation. The proposed CP helps PLLs reduce the phase offset and spurs even at ultra-low supply voltage. 